\title{
Modulation of Hypoxia in Murine Liver Metastases of Colon Carcinoma by Nicotinamide and Carbogen
}

\author{
Author(s) :H. W M. van Laarhoven, J. Bussink, J. Lok, I. Verhagen, C. J A. Punt, A. Heerschap, J. H A. \\ M. Kaanders, and A. J. van der Kogel \\ Source: Radiation Research, 164(3):245-249. 2005. \\ Published By: Radiation Research Society \\ DOI: http://dx.doi.org/10.1667/RR3435.1 \\ URL: http://www.bioone.org/doi/full/10.1667/RR3435.1
}

BioOne (www.bioone.org) is a nonprofit, online aggregation of core research in the biological, ecological, and environmental sciences. BioOne provides a sustainable online platform for over 170 journals and books published by nonprofit societies, associations, museums, institutions, and presses.

Your use of this PDF, the BioOne Web site, and all posted and associated content indicates your acceptance of BioOne's Terms of Use, available at www.bioone.org/page/terms_of_use.

Usage of BioOne content is strictly limited to personal, educational, and non-commercial use. Commercial inquiries or rights and permissions requests should be directed to the individual publisher as copyright holder. 


\title{
Modulation of Hypoxia in Murine Liver Metastases of Colon Carcinoma by Nicotinamide and Carbogen
}

\author{
H. W. M. van Laarhoven, ${ }^{a, 1}$ J. Bussink,${ }^{b}$ J. Lok, ${ }^{b}$ I. Verhagen, ${ }^{b}$ C. J. A. Punt, ${ }^{a}$ A. Heerschap, ${ }^{c}$ J. H. A. M. Kaanders ${ }^{b}$ and \\ A. J. van der $\mathrm{Kogel}^{b}$ \\ ${ }^{a}$ Departments of Medical Oncology, ${ }^{b}$ Radiation Oncology, and ${ }^{c}$ Radiology, University Medical Centre Nijmegen, 6500 HB Nijmegen, \\ the Netherlands
}

van Laarhoven, H. W. M., Bussink, J., Lok, J., Verhagen, I., Punt, C. J. A., Heerschap, A., Kaanders, J. H. A. M. and van der Kogel, A. J. Modulation of Hypoxia in Murine Liver Metastases of Colon Carcinoma by Nicotinamide and Carbogen. Radiat. Res. 164, 245-249 (2005).

There is increasing evidence that modulation of tumor hypoxia may improve therapy outcome. However, most preclinical data are derived from subcutaneous rather than orthotopic tumor models. We investigated the effect of the hypoxiamodulating agents nicotinamide and carbogen on tumor hypoxia, tumor blood perfusion, and proliferative activity in liver metastases of the murine colon carcinoma line C26a. In untreated C26a liver metastases, we observed a considerable amount of hypoxia, similar to the amount in liver metastases of patients with colorectal cancer. Compared to untreated mice, we observed a significantly smaller hypoxic fraction in the liver metastases of mice treated with nicotinamide and carbogen breathing as single treatments or in combination. In the group of mice that underwent carbogen breathing, perfusion was significantly lower than in the untreated group, but the decrease was only marginal. The proliferative activity was similar in all groups. In C26a subcutaneous tumors, a similar effect on hypoxia has been observed that was, however, combined with a decrease in proliferative activity. The different effects of nicotinamide and carbogen on parameters of the tumor microenvironment in liver metastases and subcutaneous tumors suggest that the host tissue influences the mechanism by which nicotinamide and carbogen exert their effects. Since tumor hypoxia may be a clinical problem in colorectal liver metastases, our results open possibilities for further research on the effect of hypoxia modifiers on colorectal liver metastases to improve therapy outcome. 2005 by Radiation Research Society

\section{INTRODUCTION}

It is well known that tumor hypoxia may decrease the efficacy of both radiotherapy and chemotherapy (1), and

${ }^{1}$ Address for correspondence: Department of Medical Oncology 550, Radboud University Nijmegen Medical Centre, PO Box 9101, 6500 HB Nijmegen, the Netherlands; e-mail: h.vanlaarhoven@onco.umcn.nl. there is growing evidence that modulation of tumor hypoxia may improve treatment outcome (2). Preclinical studies on modulation of tumor hypoxia and other parameters of the tumor microenvironment such as vascularization, perfusion and proliferative activity have been performed mostly on subcutaneous tumors. However, several studies, particularly on angiogenesis, have shown that the characteristics of the host tissue may influence the tumor microenvironment (3). Therefore, modulation of the microenvironment by nicotinamide and carbogen may have different effects in subcutaneous tumors and at sites of metastases. Recently, we showed that tumor hypoxia in subcutaneous murine colon carcinomas can be modified by administration of nicotinamide and/or breathing of carbogen (4). The liver is the most common site for metastases of colorectal cancer, and we have demonstrated extensive hypoxia in these colorectal liver metastases (van Laarhoven et al., unpublished data). Modulation of the tumor microenvironment of liver metastases may therefore have important clinical consequences. To assess the prospects of modulating the tumor microenvironment, we investigated the effect of nicotinamide and carbogen in liver metastases of a murine colon carcinoma line.

\section{MATERIALS AND METHODS}

Murine Liver Metastasis Model

C26a cells were acquired from Dr. G. J. Peters of the Vrije Universiteit (Amsterdam, the Netherlands) and grown in DMEM $+10 \%$ FCS. A total of 100,000 cells were injected in the spleen of female BALB/c mice; after 1 day, the spleen was removed to avoid local tumor growth. Animals were kept according to institutional guidelines for animal care. Experiments were performed 15 days after injection of the cells. Pilot experiments had shown that at that time multiple metastases had developed in the liver. All experiments were approved by the institutional ethical committee for animal use.

\section{Nicotinamide and Carbogen}

Nicotinamide (Sigma-Aldrich) $(20 \mathrm{mg} / \mathrm{ml})$ was injected intraperitoneally (ip) at a concentration of $500 \mathrm{mg} / \mathrm{kg}$. For carbogen $\left(95 \% \mathrm{O}_{2}, 5 \%\right.$ $\mathrm{CO}_{2}$, Hoek Loos, Schiedam, the Netherlands) breathing, the mice were put in a Perspex box with a continuous carbogen flow of 5 liters $/ \mathrm{min}$. Nicotinamide was administered $20 \mathrm{~min}$ before the start of carbogen 
breathing. Carbogen breathing continued for $30 \mathrm{~min}$ before the animals were killed. The mice were treated with nicotinamide alone $(n=4)$, carbogen alone $(n=5)$, or the combination of both $(n=5)$. The effects on tumor hypoxia, perfusion and proliferation were analyzed and compared with a control group of animals kept under normal air breathing $(n$ $=4)$.

\section{Markers of Hypoxia, Perfusion and Proliferation}

Pimonidazole [1-((2-hydroxy-3-piperidinyl)propyl)-2-nitroimidazole hydrochloride, Natural Pharmacia International, Belmont, CA] (5-7), which is a bio-reductive chemical probe with an immuno-recognizable side chain, was used as a marker of hypoxia. Pimonidazole $(20 \mathrm{mg} / \mathrm{ml})$ was combined with the perfusion marker Hoechst 33342 (Serva, Heidelberg, Germany) $(3.75 \mathrm{mg} / \mathrm{ml})$, and $0.1 \mathrm{ml}$ of this solution was injected intravenously in the tail vein of each mouse. As a marker of proliferation, the S-phase marker bromodeoxyuridine (BrdUrd) (Sigma-Aldrich, Zwijndrecht, the Netherlands) $(2.5 \mathrm{mg} / \mathrm{ml})$ was injected ip at a dose of $0.5 \mathrm{ml}$. Pimonidazole/Hoechst and BrdUrd were injected $25 \mathrm{~min}$ before the animals were killed by cervical dislocation. This time was chosen because the maximum effect of nicotinamide on the blood perfusion has been described $20 \mathrm{~min}$ after ip injection of nicotinamide (8), and for carbogen the maximum effect on $p \mathrm{O}_{2}$ has been observed after 5 min of carbogen breathing (9). Livers were removed immediately, cut in fragments of approximately $6 \mathrm{~mm}$, and stored in liquid nitrogen. Frozen sections $5 \mu \mathrm{m}$ thick were cut for staining and further analysis.

\section{Immunohistochemical Staining of Murine Liver Metastases}

After thawing, the sections were fixed in cold $\left(4^{\circ} \mathrm{C}\right)$ acetone for 10 min. Then slides were scanned (see below) for the Hoechst 33342 signal before immunohistochemical staining of the other markers. After scanning of the Hoechst 33342 signal and between all consecutive steps of the staining procedure, sections were rinsed three times for $2 \mathrm{~min}$ in PBS. Sections stained for pimonidazole were mounted in Fluorostab (Organon, Boxtel, the Netherlands), and the sections stained for BrdUrd were mounted in phosphate-buffered saline (PBS). The first tissue section was stained for endothelium plus the hypoxia marker pimonidazole. The second consecutive tissue section was stained for endothelium and the Sphase marker BrdUrd.

Endothelial structures and pimonidazole. After rehydration for $20 \mathrm{~min}$ in PBS, sections were incubated with rabbit anti-pimonidazole (J. A. Raleigh), diluted 1:800 in polyclonal liquid diluent (PLD, Euro-DPC, Breda, the Netherlands), for $45 \mathrm{~min}$ at $37^{\circ} \mathrm{C}$. After incubation with donkey antirabbitF $\left(\mathrm{ab}^{\prime}\right)_{2}$-Alexa488 (Molecular Probes, Leiden, The Netherlands), diluted 1:400 in PLD for $45 \mathrm{~min}$ at $37^{\circ} \mathrm{C}$, sections were incubated with undiluted 9F1, a rat monoclonal antibody to mouse endothelium, for 60 min at room temperature [Department of Pathology, Radboud University Nijmegen Medical Center, the Netherlands (10)]. Finally, sections were incubated for $30 \mathrm{~min}$ at $37^{\circ} \mathrm{C}$ with chicken anti-rat-Alexa647 (Molecular Probes) diluted 1:200 in PLD.

Endothelial structures and BrdUrd. DNA was denatured by incubation for $10 \mathrm{~min}$ at room temperature in $2 M$ hydrochloric acid. The $\mathrm{pH}$ was neutralized by rinsing the sections for $10 \mathrm{~min}$ in $0.1 \mathrm{M}$ borax. Then sections were incubated for $60 \mathrm{~min}$ at $37^{\circ} \mathrm{C}$ with sheep anti-BrdUrd (Abcam Ltd, Cambridge, UK) diluted 1:50 in PLD overnight at $4^{\circ} \mathrm{C}$. Then, sections were incubated with donkey anti-sheep-Cy3 (Jackson Immuno Research Laboratories) diluted 1:400 in PLD for $30 \mathrm{~min}$ at $37^{\circ} \mathrm{C}$. After incubation with $9 \mathrm{~F} 1$ for $60 \mathrm{~min}$ at room temperature, sections were incubated $30 \mathrm{~min}$ at $37^{\circ} \mathrm{C}$ with chicken anti-rat-Alexa647 (Molecular Probes) diluted 1:200 in PLD. Finally, sections were incubated with Fast Blue diluted 1:1000 in PBS for $5 \mathrm{~min}$ at room temperature.

\section{Image Analysis}

Quantitative data for tumor hypoxia, perfusion and proliferation were acquired with a semiautomatic method based on a computerized digital image analysis system that has been described previously and has been shown to be an accurate and reproducible technique (11-13). Briefly, we used a high-resolution intensified solid-state camera on a fluorescence microscope (Axioskop, Zeiss, Weesp, the Netherlands) with a computercontrolled motorized stepping stage to scan whole tumor cross sections sequentially, with different filters for the detection of the fluorescence signals. Analysis of hypoxia and vascular structures was performed with $100 \times$ magnification, and analysis of proliferation was performed with $200 \times$ magnification. Depending on the size of the tumor section, each scan consisted of 36-144 fields of $1.2 \mathrm{~mm}^{2}$. One composite image was reconstructed from the individual microscopic fields. A contour line was drawn to delineate the tumor area, thereby excluding non-tumor tissue (such as normal liver tissue and necrosis) from the analyses, using consecutive H\&E-stained tumor sections. The hypoxic fraction of the tumor section was computed as the tumor area stained by the hypoxia marker relative to the total viable tumor area. The perfused fraction of the tumor was defined as the area that was stained by both Hoechst 33342 and 9F1 (i.e. perfused vascular area) relative to the total vascular area. The relative vascular area was calculated by dividing the 9F1-positive area by the total viable tumor area. The BrdUrd labeling index was calculated as the ratio of the BrdUrd-positive area and the total nuclear area.

\section{Statistics and Calculations}

The $t$ test for independent samples was used to compare differences in hypoxic fraction, perfused fraction, relative vascular area and labeling index between different groups of mice.

\section{RESULTS}

In all mice, several metastases of about $2 \mathrm{~mm}$ were observed in the liver. Figure 1 shows a small part of the liver containing three metastases. No preference for a specific lobe of the liver was noted, and no metastases were seen in other organs. The metastases consisted of densely packed tumor cells and stroma without clear structural organization (Fig. 1A). In untreated mice, areas of hypoxia were found throughout the metastases (Fig. 1B).

Microscopic examination showed that, compared to the control group, less hypoxia was present in the liver metastases that were treated with nicotinamide, carbogen or the combination of nicotinamide and carbogen (Fig. 2). For a quantitative analysis of hypoxia and vasculature, two or three fragments of the liver per mouse were analyzed separately; for a quantitative analysis of the BrdUrd labeling index, one fragment of the liver per mouse was used. Results of the quantitative analysis are shown in Table 1. A significant decrease in hypoxic fraction was observed after treatment with nicotinamide, carbogen and the combination of nicotinamide and carbogen $(P<0.05$; Fig. 3). Although a significantly lower perfused fraction was observed in the group of mice that underwent carbogen breathing compared to the control group $(P<0.05)$, the decrease in the perfused fraction was only marginal, from 0.89 in control animals to 0.83 in carbogen-treated animals. The relative vascular area and BrdUrd labeling index were not significantly different in the treated groups compared to the control group. 

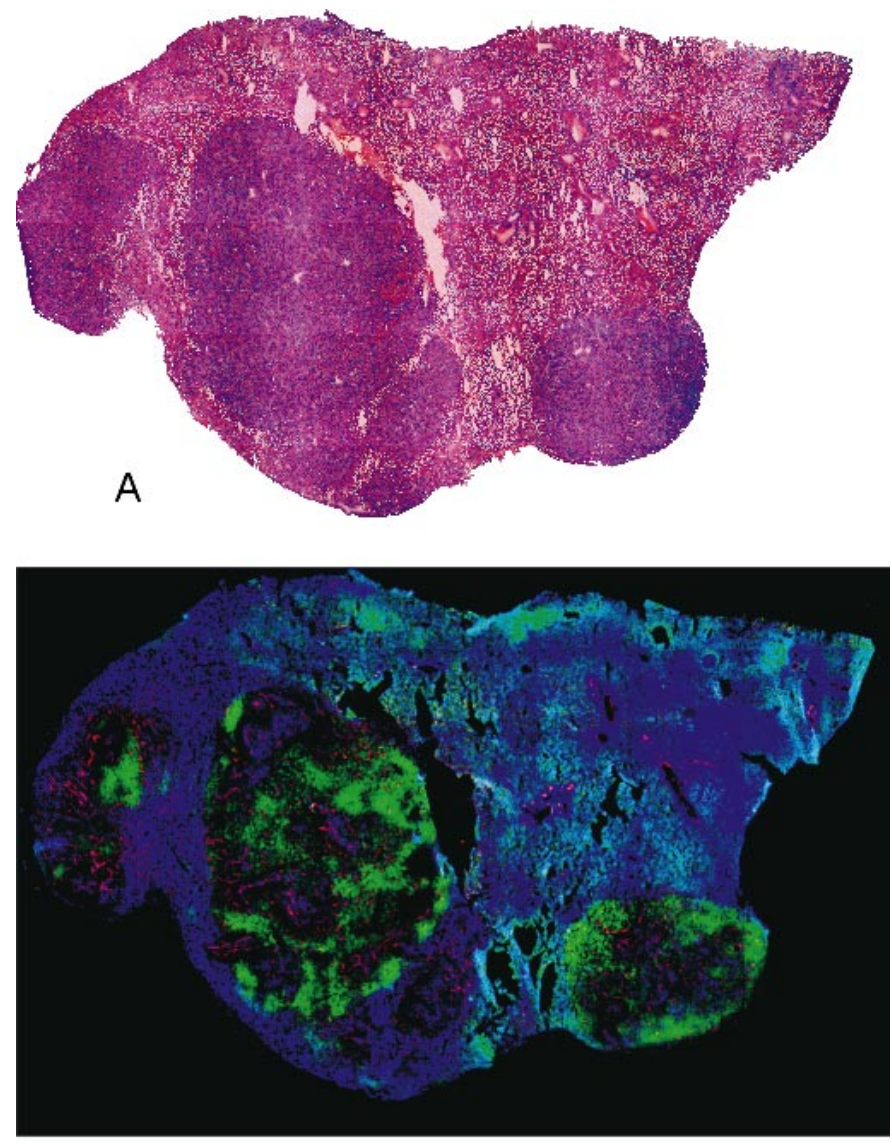

B

FIG. 1. Panel A: H\&E scan of part of the liver of one mouse from the control group that contained three liver metastases of C26a tumor. Panel B: The corresponding liver metastases stained for hypoxia as indicated by pimonidazole binding (green), perfusion (blue) and vasculature (red-pink). The scale bar indicates $1 \mathrm{~mm}$. Original magnification $100 \times$.

\section{DISCUSSION}

In untreated C26a liver metastases, we observed a hypoxic fraction of 0.12 , which is similar to the amount of hypoxia in liver metastases of patients with colorectal cancer (van Laarhoven et al., unpublished data). Recently, we described hypoxia, vascularity and proliferative activity of subcutaneous C26a tumors implanted in the flank of BALB/ c mice and the response to nicotinamide and carbogen (4). Quantitative data for hypoxia and proliferative activity were similar for the liver metastases and subcutaneous tumors of the same tumor line. However, a larger relative vascular area and a higher perfused fraction were observed in the liver metastases compared to subcutaneous tumors (relative vascular area: 0.05 compared to 0.02 and perfused fraction: 0.89 compared to 0.56 , respectively, $P<0.05$ ). Although the time between the administration of Hoechst dye and freezing of the tumors was relatively long ( $25 \mathrm{~min}$ ), we have established in this tumor model that the Hoechst dye does not diffuse further than a few cell layers from the vessel and does not influence the measured perfused fraction. This time was chosen to monitor the distribution of pimonidazole, which needs to be administered a minimum of $25 \mathrm{~min}$ before animals are killed to allow for binding to hypoxic cells. Since this time was used in both liver metastases and subcutaneous tumors, it cannot explain the large perfused fraction in liver metastases compared to subcutaneous tumors. Since liver metastases were smaller than subcutaneous tumors, it could be hypothesized that when tumor growth is faster than growth of (functional) vasculature, the perfused fraction decreases at larger tumor sizes. Alternatively, the host vasculature may play a role in the difference in perfused fraction between liver metastases and subcutaneous tumors. The vasculature of primary tumors and metastases may consist of neoangiogenic vessels or of host vessels that have been co-opted by the tumor (14). If

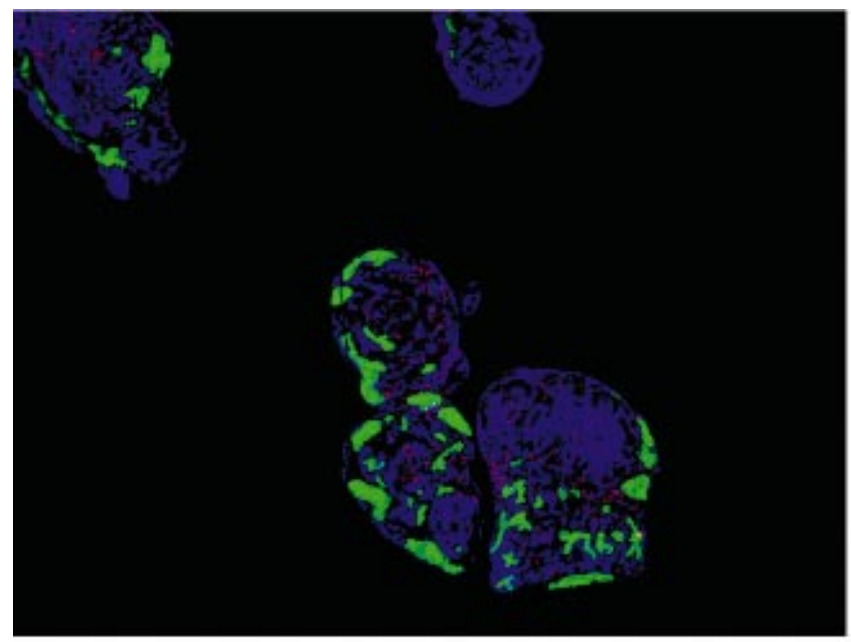

A

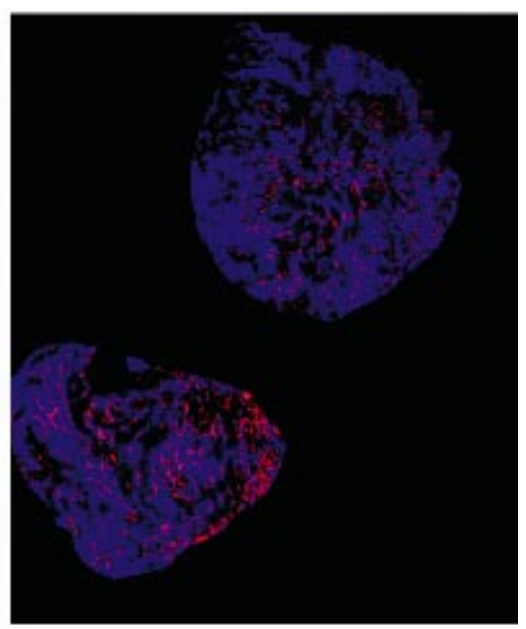

B

FIG. 2. Binary maps of complete sections of C26a colon liver metastases without treatment (panel A) and after nicotinamide administration combined with carbogen breathing (panel B). Vascular structures: red-pink; perfused vessels: blue; hypoxic areas: green. The scale bars indicate $100 \mu \mathrm{m}$. Original magnification $100 \times$. 
TABLE 1

Average Hypoxic Fraction, Perfused Fraction, Relative Vascular Area, and BrdUrd Labeling Index \pm SD for all Liver Fragments of the Control and Treatment Groups

\begin{tabular}{lcccc}
\hline \multicolumn{1}{c}{ Treatment } & Hypoxic fraction & Perfused fraction & $\begin{array}{c}\text { Relative } \\
\text { vascular area }\end{array}$ & Labeling index \\
\hline Control & $0.12 \pm 0.11$ & $0.89 \pm 0.05$ & $0.05 \pm 0.02$ & $0.06 \pm 0.02$ \\
Nicotinamide & $0.01 \pm 0.01^{* *}$ & $0.89 \pm 0.07$ & $0.04 \pm 0.02$ & $0.13 \pm 0.07$ \\
Carbogen & $0.03 \pm 0.03^{*}$ & $0.83 \pm 0.07 *$ & $0.05 \pm 0.02$ & $0.09 \pm 0.06$ \\
Nicotinamide + carbogen & $0.02 \pm 0.04^{*}$ & $0.87 \pm 0.10$ & $0.04 \pm 0.02$ & $0.09 \pm 0.04$ \\
\hline
\end{tabular}

Notes. Two to three sections from different locations in the liver per mouse from four or five mice were used.

$* P<0.05$ compared with the control group; ** $P<0.01$ compared with the control group.

C26a tumors mainly depend on co-option for their vascularization, this would explain the larger perfused fraction in liver metastases compared to subcutaneous tumors, since the liver is an extremely well-perfused organ compared to subcutaneous tissue. In the subcutaneous tumors, we observed a hypoxic rim, possibly caused by pressure of the surrounding tissue on the small C26a tumor vessels, resulting in compression of vasculature, thereby hindering tumor blood perfusion and leading to hypoxia. In the liver metastases model used in this study, we did not observe a hypoxic rim, which is probably due to reduced tissue pressure in the liver compared with overlying skin.

To assess the feasibility of modulating tumor hypoxia, we investigated the effect of nicotinamide and carbogen in C26a liver metastases. A significant decrease in hypoxic fraction was observed after treatment with nicotinamide and carbogen as single agents as well as after the combination treatment, similar to our previous findings for subcutaneously implanted C26a tumors (4). It should be noted that except for the murine $\mathrm{C} 26 \mathrm{a}$ and $\mathrm{C} 38$ colon carcinomas, a reduction in hypoxic fraction of this magnitude after administration of nicotinamide as a single agent has not been observed in experimental tumors before.

Several mechanisms could play a role in the observed

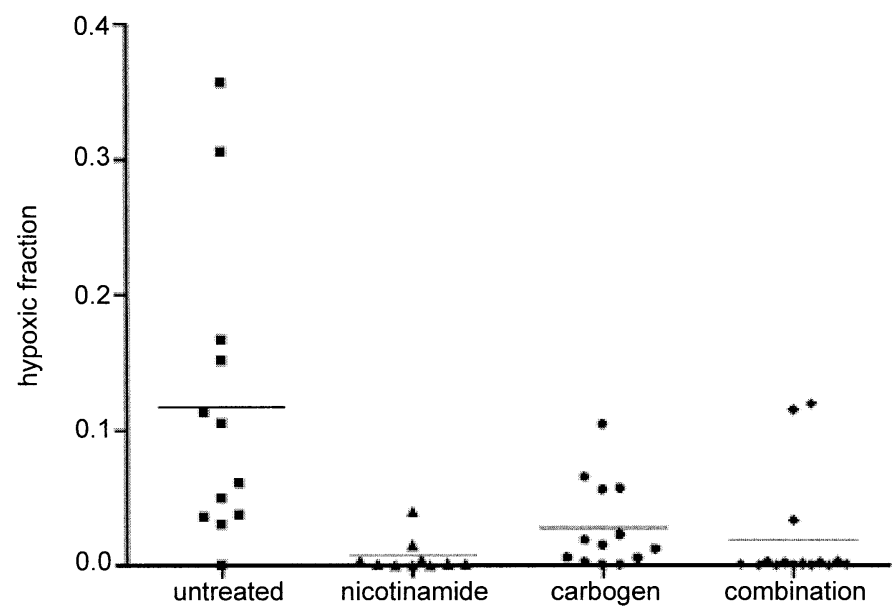

FIG. 3. Quantitative assessment of the hypoxic fraction per liver fragment in C26a liver metastases. Values are shown for control tumors and for tumors after administration of nicotinamide, carbogen and the combination of both. The horizontal line indicates the mean for each group. decrease in hypoxia in liver metastases. For nicotinamide, the classical hypothesis is that a decrease in hypoxic fraction can be attributed to an improved tumor perfusion (15) by suppression of transient fluctuations in microregional blood flow (16). Although in our experiments no increase in perfusion of the liver metastases was observed, these transient fluctuations may not have been picked up by the current protocol, where we chose an interval of $25 \mathrm{~min}$ between nicotinamide and Hoechst dye administration. Previously, we have suggested that nicotinamide may decrease hypoxia in C26a tumors by increasing blood glucose (4), possibly by induction of glycogen breakdown $(17,18)$. The so-called Crabtree effect (19), which refers to a decrease in cell respiration rate after addition of glucose, would be accompanied by a decrease in proliferative activity, due to enhanced anaerobic glycolysis, leading to an overproduction of lactic acid that may disrupt tumor development. However, in liver metastases no decrease in BrdUrd labeling index was observed, and therefore the Crabtree effect does not appear to occur. This suggests that the host tissue may have some influence on the mechanism by which nicotinamide exerts its effects.

For carbogen, it has been proposed that increased oxygen transport by the plasma causes a decrease in tumor hypoxia (20). This decrease in tumor hypoxia has also been described as being a result of the so-called vascular steal effect (21). In tumor vasculature, which lacks responsive smooth musculature and which is arranged in parallel with the host vasculature of surrounding normal tissue, carbogen breathing can cause a reduction in tumor blood perfusion. This is due to the vasodilating effect of the $\mathrm{CO}_{2}$ component of carbogen on host vessels in the surrounding normal tissues, "stealing" blood from the tumor. Hence the reduced supply of nutrients would decrease the $\mathrm{O}_{2}$ consumption rate of tumor cells and decrease the hypoxic fraction (9). However, in liver metastases, tumor vasculature, especially when it consists of co-opted host vessels, is more likely to be in series with host vasculature rather than in parallel. Therefore, it is unlikely that a vascular steal effect plays an important role in the observed decrease in hypoxic fraction.

Although the reduction in hypoxic fraction caused by nicotinamide and carbogen in liver metastases seems to occur through different mechanisms (suppression of fluctua- 
tions in blood flow and increased plasma oxygen transport, respectively), the magnitude of the effect was similar.

Since tumor hypoxia has been related to poor prognosis due to progression to a more malignant phenotype and increased resistance to therapy (22), it has become a central issue in cancer treatment. Clinical studies in several tumor types (23-26) have shown promising results with the combination of hypoxia modulation and radiation therapy. For advanced colorectal cancer, chemotherapy is often the treatment of choice. A variety of direct and indirect mechanisms have been described by which hypoxia can decrease chemotherapy efficacy (27). For example, shutdown of blood vessels (acute hypoxia) and increased diffusion distances (chronic hypoxia) diminish the distribution of chemotherapeutic agents. Other mechanisms include an increased activity of DNA repair enzymes and increased invasiveness and angiogenic potential. In this study, we observed in an orthotopic murine model that hypoxia is present in liver metastases of colon carcinoma and that hypoxia can be decreased using nicotinamide and carbogen. Further experiments will show whether the combination of these hypoxia modifiers with chemotherapy can improve the outcome of treatment of advanced colon cancer.

\section{ACKNOWLEDGMENTS}

We thank B. Lemmers, I. Elemans, G. Grutters and colleagues at the Central Animal Laboratory for biotechnical assistance and animal care. This study was supported financially by the Dutch Cancer Society, grant KUN 2000-2307.

Received: February 24, 2005; accepted: May 23, 2005

\section{REFERENCES}

1. J. M. Brown, Exploiting the hypoxic cancer cell: Mechanisms and therapeutic strategies. Mol. Med. Today 6, 157-162 (2000).

2. J. H. Kaanders, J. Bussink and A. J. van der Kogel, Clinical studies of hypoxia modification in radiotherapy. Semin. Radiat. Oncol. 14, 233-240 (2004).

3. W. L. Monsky, C. C. Mouta, Y. Tsuzuki, T. Gohongi, D. Fukumura and R. K. Jain, Role of host microenvironment in angiogenesis and microvascular functions in human breast cancer xenografts: mammary fat pad versus cranial tumors. Clin. Cancer Res. 8, 1008-1013 (2002).

4. H. W. van Laarhoven, J. Bussink, J. Lok, C. J. Punt, A. Heerschap and A. J. van der Kogel, Effects of nicotinamide and carbogen in different murine colon carcinomas: Immunohistochemical analysis of vascular architecture and microenvironmental parameters. Int. J. Radiat. Oncol. Biol. Phys. 60, 310-321 (2004).

5. R. E. Durand and J. A. Raleigh, Identification of nonproliferating but viable hypoxic tumor cells in vivo. Cancer Res. 58, 3547-3550 (1998).

6. G. E. Arteel, R. G. Thurman, J. M. Yates and J. A. Raleigh, Evidence that hypoxia markers detect oxygen gradients in liver: Pimonidazole and retrograde perfusion of rat liver. Br. J. Cancer 72, 889-895 (1995).

7. J. A. Raleigh, S. C. Chou, G. E. Arteel and M. R. Horsman, Comparisons among pimonidazole binding, oxygen electrode measurements, and radiation response in $\mathrm{C} 3 \mathrm{H}$ mouse tumors. Radiat. Res. 151, 580-589 (1999).

8. J. Bussink, J. H. Kaanders, A. M. Strik and A. J. van der Kogel, Effects of nicotinamide and carbogen on oxygenation in human tu- mor xenografts measured with luminescence based fiber-optic probes. Radiother. Oncol. 57, 21-30 (2000).

9. B. P. van der Sanden, B. P. Heerschap, A. Hoofd, L. Simonetti, K. Nicolay, K. van der Toom, W. N. Colier and A. J. van der Kogel, Effect of carbogen breathing on the physiological profile of human glioma xenografts. Magn. Reson. Med. 42, 490-499 (1999).

10. J. R. Westphal, R. G. van't Hullenaar, J. A. van der Laak, I. M. Cornelissen, L. J. Schalkwijk, G. N. van Muijen, P. Wesseling, P. C. de Wilde, D. J. Ruiter and R. M. de Waal, Vascular density in melanoma xenografts correlates with vascular permeability factor expression but not with metastatic potential. Br. J. Cancer 76, 561-570 (1997).

11. P. F. Rijken, H. J. Bernsen and A. J. van der Kogel, Application of an image analysis system to the quantitation of tumor perfusion and vascularity in human glioma xenografts. Microvasc. Res. 50, 141153 (1995).

12. J. Bussink, J. H. Kaanders, P. F. Rijken, C. A. Martindale and A. J. van der Kogel, Multiparameter analysis of vasculature, perfusion and proliferation in human tumour xenografts. Br. J. Cancer 77, 57-64 (1998).

13. J. Bussink, J. H. Kaanders, P. F. Rijken, J. A. Raleigh and A. J. van der Kogel, Changes in blood perfusion and hypoxia after irradiation of a human squamous cell carcinoma xenograft tumor line. Radiat. Res. 153, 398-404 (2000).

14. P. B. Vermeulen, C. Colpaert, R. Salgado, R. Royers, H. Hellemans, H. E. Van Den, G. Goovaerts, L. Y. Dirix and E. van Marck, Liver metastases from colorectal adenocarcinomas grow in three patterns with different angiogenesis and desmoplasia. J. Pathol. 195, 336342 (2001).

15. D. J. Chaplin, M. R. Horsman and M. J. Trotter, Effect of nicotinamide on the microregional heterogeneity of oxygen delivery within a murine tumor. J. Natl. Cancer Inst. 82, 672-676 (1990).

16. S. A. Hill and D. J. Chaplin, The effect of nicotinamide on microregional blood flow within tumours assessed using laser Doppler probes. Acta Oncol. 34, 401-404 (1995).

17. H. P. Ammon and C. J. Estler, The effect of nicotinic acid on glycolytic carbohydrate breakdown in the liver. Life Sci. 6, 641-647 (1967).

18. F. J. Moreno, L. Sanchez-Urrutia, J. M. Medina, F. Sanchez-Medina and F. Mayor, Stimulation of phosphoenolpyruvate carboxykinase (guanosine triphosphate) activity by low concentrations of circulating glucose in perfused rat liver. Biochem. J. 150, 51-58 (1975).

19. H. G. Crabtree, Observations on the carbohydrate metabolism of tumours. Biochem. J. 23, 536-545 (1929).

20. M. E. Powell, S. A. Hill, M. I. Saunders, P. J. Hoskin and D. J. Chaplin, Effect of carbogen breathing on tumour microregional blood flow in humans. Radiother. Oncol. 41, 225-231 (1996).

21. R. L. Jirtle, Chemical modification of tumour blood flow. Int. J. Hyperthermia 4, 355-371 (1988).

22. P. Vaupel, A. Mayer and M. Hockel, Tumor hypoxia and malignant progression. Methods Enzymol. 381, 335-354 (2004).

23. J. H. Kaanders, L. A. Pop, H. A. Marres, J. Liefers, F. J. van den Hoogen, W. A. van Daal and A. J. van der Kogel, Accelerated radiotherapy with carbogen and nicotinamide (ARCON) for laryngeal cancer. Radiother. Oncol. 48, 115-122 (1998).

24. P. J. Hoskin, M. I. Saunders, H. Phillips, H. Cladd, M. E. Powell, K. Goodchild, M. R. Stratford and A. Rojas, Carbogen and nicotinamide in the treatment of bladder cancer with radical radiotherapy. $B r . J$. Cancer 76, 260-263 (1997).

25. M. J. Droller, Hypoxic radiosensitizers in radical radiotherapy for patients with bladder carcinoma: Hyperbaric oxygen, misonidazole, and accelerated radiotherapy, carbogen and nicotinamide. J. Urol. 163, 1600 (2000).

26. K. Pigott, S. Dische and M. I. Saunders, Short communication: The addition of carbogen and nicotinamide to a palliative fractionation schedule for locally advanced breast cancer. Br. J. Radiol. 68, 215218 (1995).

27. L. Harrison and K. Blackwell, Hypoxia and anemia: factors in decreased sensitivity to radiation therapy and chemotherapy? Oncologist 9 (Suppl.), 31-40 (2004). 